\title{
Association of Serum Vitamin D Status with gestational diabetes mellitus and other laboratory parameters in early pregnant women
}

Caihong Luo ( $\sim$ hym526526@163.com )

Foshan Women's and Children's Hospital of Sothern Medical University

\section{Zhiju Li}

Southern Medical University

\section{Yunya Lu}

Foshan Women's and Children's Hospital of Sothern Medical University

\section{Fang Wei}

Foshan Women's and Children's Hospital of Sothern Medical University

\section{Dongmei Suo}

Foshan Women's and Children's Hospital of Sothern Medical University

\section{Shiyan Lan}

Foshan Women's and Children's Hospital of Sothern Medical University

\section{Zhengyuan Ren}

Foshan Women's and Children's Hospital of Sothern Medical University

\section{Runchang Jiang}

Foshan Women's and Children's Hospital of Sothern Medical University

\section{Fang Huang}

Foshan Women's and Children's Hospital of Sothern Medical University

\section{Aiyue Chen}

Foshan Women's and Children's Hospital of Sothern Medical University

\section{Liejun Jiang}

the People's Hospital of Guangxi Zhuang Autonomous Region

\section{Huayi Huang}

Roswell Park Cancer Institute

\section{Xiaoling Guo}

Foshan Women's and Children's Hospital of Sothern Medical University

\section{Research Article}

Keywords: 25-hydroxy vitamin D (VD), gestational diabetes mellitus (GDM), alkaline phosphatase, prealbumin, thrombin time 
Posted Date: February 15th, 2021

DOl: https://doi.org/10.21203/rs.3.rs-189644/v1

License: (c) (i) This work is licensed under a Creative Commons Attribution 4.0 International License. Read Full License 


\section{Abstract}

Background: Pregnant women develop certain metabolic alterations and substantial nutrient insufficiency, which may impact fetus development. Reports on the association of serum 25-hydroxy vitamin D (25(OH)D, VD) status with gestational diabetes mellitus (GDM) is still controversial. This study aimed to investigate the association of serum VD status with GDM and other laboratory parameters in early pregnant women.

Methods: A total of 1516 pregnant women were enrolled in this study. Maternal serum VD and other laboratory parameters-including hematology, coagulation, chemistry, and bone density-were measured utilizing various analytical methods in clinical laboratory at gestational weeks 11 to 14 . Serum VD level was subsequently correlated with blood glucose and other laboratory parameters.

Results: The average VD concentration was $59.12 \pm 12.5 \mathrm{nmol} / \mathrm{L}$. None of the study subjects had VD <25 $\mathrm{nmol} / \mathrm{L} ; 434$ (28.6\%) women had VD $<50 \mathrm{nmol} / \mathrm{L}$, and 200 women (13.2\%) had VD $\geq 75 \mathrm{nmol} / \mathrm{L}$. There were 264 (17.4\%) women diagnosed with GDM. There was not, however, a correlation between serum VD and GDM in early pregnancy. Interestingly, women with more parity and high serum alkaline phosphatase levels had higher serum VD levels. There was a positive correlation between serum VD and pre-albumin, activated partial thromboplastin time, and a negative correlation between serum VD, creatinine, and thrombin time.

Conclusions: There was no correlation between maternal serum VD and GDM in early pregnant women. There were, however, associations between serum VD and parity, serum alkaline phosphatase, creatinine, pre-albumin, and coagulation factors, which need further study to explain their pathophysiology and clinical significance.

\section{Background}

Vitamin $\mathrm{D}(\mathrm{VD})$ is a fat-soluble vitamin, which plays an important role in bone mineralization, calcium and phosphorus absorption, parathyroid status and immune system functionality [1]. In the human body, VD from sunlight and food is hydroxylated to 25-hydrovitamin D (25(OH)D) in the liver and is subsequently activated with a second hydroxylation to 1,25-dihydroxy vitamin $D$ in the kidney [2].

Study found that during pregnancy, calcitriol increases in early gestation to meet a mother's needs, and maternal serum 25(OH)D crosses into the placenta for use in fetus development [3]. Abnormal VD levels may cause some adverse pregnancy complications, including polycystic ovary syndrome, gestational diabetes mellitus (GDM), pre-eclampsia, infertility, endometriosis, and cancers [4-7]. Studies on the association between VD status and pregnancy complications are still controversial. On the one hand, some studies indicated that the alteration of VD was associated with a variety of adverse pregnancy outcomes, especially GDM [8-10]. On the other hand, some studies failed to correlate VD status with the above-mentioned consequences [11,12]. 
In this study, the association between maternal serum VD levels and GDM and other laboratory parameters in early pregnancy were observed to help better understand metabolic events and their potential effects on fetus development, and the potential diagnostic and prognostic applications.

\section{Patients And Methods}

\section{Patient enrollment and ethics approval}

A total of 1516 women with ages from 18 to 46 were recruited from August 1, 2018 to August 31, 2019 at Foshan Women and Children's Hospital of Southern Medical University, which is a specialized tier-3, grade-A hospital in South China. Pregnant women with singleton pregnancies and conceiving naturally were included. The exclusion criteria included pre-existing diabetes, hypertension or other chronic diseases, abnormal child-bearing history, a history of drug use, and vitamin supplements-including vitamin D and calcium-that may affect metabolism or unavailable data. All pregnant women exerted a normal daily life without special sunlight exposure or quarantines. All the study subjects were living in the in the Foshan, Guangdong Province of South China. Ethics approval was obtained by the Institutional Review Board (IRB) and Ethics Committee of Foshan Women and Children's Hospital of Southern Medical University. All methods were carried out in accordance with relevant guidelines and regulations. Methods and laboratory testing are routine and standard operating required for medical practice in Guangdong Province including this hospital. All participants provided written informed consent.

\section{Serum vitamin $D$ analysis}

Serum VD was analyzed at gestational weeks 11 to 14 using a Mokosensor-A300 immune colloidal gold analyzer (MedicalSystems, Ningbo, China) following the manufacturer's instructions.

\section{Chemistry analysis}

Fasting and $1 \mathrm{~h}, 2 \mathrm{~h}$ oral glucose tolerance test (OGTT) blood glucose, alanine aminotransferase, alkaline phosphatase, pre-albumin, creatinine, cystatin C, calcium, and magnesium were analyzed on a BeckmanCoulter Automatic Chemistry Analyzer platform (Beckman-Coulter, Brea, CA, USA) following the manufacturer's instructions and laboratory operating procedures. The collection of blood samples, including the selection of a collecting tube, was followed by routine clinical laboratory procedures.

\section{Glucose tolerance test}

A $75 \mathrm{~g}$ oral glucose tolerance test ( $75 \mathrm{~g}$ OGTT) was performed between 24 and 28 weeks of gestation. Either of the following criteria met was diagnosed as GDM: fasting serum glucose $\geq 5.1 \mathrm{mmol} / \mathrm{L}, 1 \mathrm{~h}$ of OGTT serum glucose $\geq 10 \mathrm{mmol} / \mathrm{L}$, or $2 \mathrm{~h}$ of $\mathrm{OGTT}$ serum glucose $\geq 8.5 \mathrm{mmol} / \mathrm{L}$.

\section{Hematology analysis}


Hematology analysis was performed on a Sysmex XN2000 hematology analyzer platform (Sysmex, Kobe, Japan), and hematological parameters, including the indices, were obtained.

\section{Coagulation test}

The blood coagulation test was performed on a Stago STA-R Evolution (Diagnostica Stago, Asnières sur Seine Cedex, France) platform following the manufacturer's instructions using a heparin anti-coagulant blood collection tube.

\section{Bone density examination}

Bone density was measured using a Hong Yang BMD-1000 Ultrasound Bone Sonometer (Baoding, Hebei, China) following the manufacturer's instructions.

\section{Statistical Analysis}

Statistical analysis was performed using the SPSS (version 24). Independent-samples t-test, One-Way ANOVA, and Pearson's correlation were used in the analysis. A $P<0.05$ were considered statistically significant.

\section{Results}

\section{Basic information of study subjects}

Table 1 lists the basic information of study subjects in this study.

\section{Association between maternal serum vitamin D level and gestational diabetes mellitus}

Serum VD level was correlated with maternal fasting, OGTT 1h, $2 \mathrm{~h}$ blood (serum) glucose levels. VD was also correlated with GDM and non-GDM status of pregnant women. As shown in Table 2, there is no association between VD and glucose levels in early gestation women ( $p>0.05$ for all).

\section{Correlation of serum vitamin D level with age, blood chemistry, coagulation, and trace elements} parameters

While correlating chemistry parameters with VD level in serum, results showed that there was no association between serum VD and age, alanine aminotransferase (ALT), cystatin C, calcium, and magnesium, as shown in Table 3 ( $p>0.05$ for all).

\section{Correlation of serum vitamin D with hematologic parameters}

Serum VD level was also correlated with hematologic parameters of early pregnant women. Table 4 shows that there is no association between serum VD level and red blood cells (RBC), hemoglobin (HGB), white blood cells (WBC) and neutrophils (Neu, \%), and platelet (PLT) ( $p>0.05$ for all). 


\section{Correlation of serum vitamin D with parity}

Serum VD level in pregnant women was further correlated with parity number. Table $5 \mathrm{a}$ and $5 \mathrm{~b}$ shows that women going into birth 3 times had a higher VD level than those with 2 or 1 paritys $(p=0.016$ and $p=0.039$, respectively).

\section{Association between serum vitamin D level and alkaline phosphatase activities}

There was a positive correlation between serum VD level and alkaline phosphatase activities, of which a higher serum VD level tended to have higher alkaline phosphatase activities (Table 6a and 6b, $p=0.005$ and 0.004 , respectively).

\section{Correlation of serum vitamin D with bone density, body weight of newborns, coagulation, and other chemistry parameters (Pearson's correlation)}

Furthermore, maternal serum VD was correlated with bone density, the body weight of newborns, coagulation, and other chemistry parameters besides those mentioned above. Table 7 shows that serum VD level had a positive correlation with activated partial thromboplastin time (aPTT) $(p=0.047)$, prealbumin ( $p=0.001)$, and a negative correlation with thrombin time $(T T)(p=0.000)$, creatinine $(p=0.006)$. There was no association between serum VD level and bone density, body weight of newborns, D-Dimer, and fibrinogen ( $p>0.05$ for all) (Table 7).

\section{Discussion}

The association between maternal serum VD and diabetes in early pregnancy is still controversial $[13,14]$. GDM was the most common complication of pregnancy with a prevalence of $1 \%$ to $>30 \%$ globally [15]. With excessive peripheral insulin resistance and insufficient insulin production, elevation of blood glucose increases the risk of short-term and long-term consequences in both mother and infant, including preeclampsia, macrosomia, cardiovascular disease and obesity [16]. Previous studies found that VD deficient rats had lower insulin secretion than VD deficient rats supplemented with VD [17]. A metaanalysis of 29 observational studies indicated that VD insufficiency was related to increased risk of GDM [18]. Similarly, high serum 25(OH)D in early pregnancy protects from the development of GDM [19].

The results from this study indicated that there was no association between maternal serum VD level and GDM (Table 2). This result is similar with the report from a previous meta analysis [20]. A randomized controlled trial also showed a limitation of VD supplementation for GDM prevention in VD sufficient populations [21]. Since VD level is affected by several factors, such as seasons, geographical location, race, diet/lifestyle, medical conditions, etc., it is difficult to perform quality control and draw comparisons among studies. In addition, the lack of standardized measurement methods is another concern. Thus, a consensus on VD study is needed for guiding reliable VD related studies in the future.

Growing evidence has indicated that serum VD deficiency may lead to other pregnancy adverse outcomes, such as macrosomia, pre-eclampsia, and other health issues [22,23]. A previous study, 
however, demonstrated that VD supplementation hardly reduced the risk of pre-eclampsia, preterm birth, and low body weight [24]. This study's results suggested that there was no association between maternal VD level and age ( $<=35$ vs $>35$ ), serum alanine aminotransferase (ALT), cystatin C, calcium, and magnesium (Table 3). Serum VD level was then further correlated with maternal hematological parameters red blood cells (RBC), hemoglobin (HGB), white blood cells (WBC) and the percentage of neutrophils (Neu, \%), and platelets (PLT). However, the statistical results were all insignificant (Table 4).

In this study, we found that there was a positive correlation between maternal serum VD level and the number of parity; women who had given birth 3 times had higher serum VD than those giving birth 2 or 1 time (s) ( $p=0.016$ and 0.039 , respectively, Table $5 a$ and $5 b)$. One study mentioned that there was no significant difference between the serum VD level and age, parity, or gestational age [25]. The mechanism underlying this phenomenon is therefore unclear; there could be certain compensation in metabolism function during pregnancy that requires further investigation in the future.

Our results also indicated that there was a positive correlation between serum VD and alkaline phosphatase (ALP) activity; women who had high serum VD also had higher ALP activity (Table 6a and 6b). A study observed that there was an association between ALP and moderate VD deficiency group; however, the correlation was not significant [26]. Another study also found that there was no correlation between ALP and vitamin status in school children [27].

Serum ALP is widely used in clinical practice as a marker of bone turnover [28]. Medical conditions with osteolysis had elevated serum ALP levels [29]. Based on our results, we hypothesize that there is a relationship between VD and ALP in maintaining the bone and mineral metabolic homeostasis, a hypothesis that merits further investigation.

Lastly, our study also found that there was a positive correlation between serum VD level and thrombin time (TT) measurement in pregnant women, which means that VD had an effect on blood coagulation. A previous study showed that treatment with VD supplementation had a prothrombotic effect in patients with VD insufficiency, an issue that requires further study [30]. We also observed, however, that there was a positive correlation between maternal serum VD level and activated partial thrombin time (aPTT) (Table 7). The mechanism underlying this phenomenon is unclear. We hypothesize that VD plays a role in serum calcium turnover, leading to the postpone of aPTT which requires further investigation. Our results also indicated that there was a negative correlation between serum VD and creatinine level, a positive correlation between serum VD and pre-albumin levels in pregnant women, which suggests that VD plays a role in creatinine clearance and enhancing albumin production (Table 7).

\section{Conclusion}

This study did not show an association between maternal serum VD level and GDM. There was a positive correlation between serum VD and parity, serum alkaline phosphatase, activated partial thrombin time, pre-albumin levels, and a negative correlation between serum VD and thrombin time and serum creatinine levels, which could have potential clinical significance and requires further investigation. 


\section{Declarations}

Acknowledgments: None.

Author contributions: CL, MD: principle investigator and conception; ZL, MD and LJ, MS: statistical analysis; YL, MD, FW, MD, DS, MD, SL, MD, and AC, BS: patients' enrolment and clinical information collection; ZR, BS: data collection and integration; RJ, BS: laboratory analysis and data collection; FH, BS: Bone Densitometry; $\mathrm{HH}, \mathrm{MD}, \mathrm{PhD}$ : manuscript writing; $\mathrm{XL}, \mathrm{MD}$ : project organization and conception.

Funding: Foshan Science and Technology Bureau Project (2018AB000251) of Guangdong, China

\section{Availability of data and materials}

Data is available from the corresponding author upon inquiry.

\section{Ethics approval and consent to participate}

Ethics approval was obtained by the Institutional Review Board (IRB) and Ethics Committee of Foshan Women's and Children's Hospital of Southern Medical University. The approvel document number is FSFYMEC-2018-014, the file will be provided upon inquiring. All experimental protocols were approved by the IRB of the hospital. All methods were carried out in accordance with relevant guidelines and regulations. Methods and laboratory testing are routine and standard operating required for medical practice in Guangdong Province including this hospital. All participants provided written informed consent.

\section{Consent for publication}

Not applicable.

\section{Competing interests}

The authors declare no conflict of interest.

\section{Author details}

1 Department of Obstetrics, The Women's and Children's Hospital of Southern Medical University, Foshan, Guangdong, China; hym526526@163.com (C.L.); luyy397@163.com (Y.L.); strawberry1106@163.com (F.W.); fssdm@163.com (D.S.); Isywait@sina.com (S.L.); 282069560@qq.com (F.H.); 1353139944@qq.com (A.C.); fsguoxl@163.com (X.G.)

2 Department of Epidemiology, School of Public Health, Southern Medical University, Guangzhou, Guangdong, China; 597105587@qq.com (Z.L.)

3 Department of Information Technology, The Women's and Children's Hospital of Southern Medical University, Foshan, Guangdong, China; 275533129@qq.com (Z.R.) 
4 Department of Laboratory Medicine, The Women's and Children's Hospital of Southern Medical University, Foshan, Guangdong, China; 745717198@qq.com (R.J.)

5 Department of Laboratory Medicine, the People's Hospital of Guangxi Zhuang Autonomous Region, Nanning, Guangxi, China; 2529767940@qq.com (L.J.)

6,7,8 School of Medical Laboratory, Youjiang Medical University for Nationalities, No. 98 Chengxiang Road, Baise, Guangxi 533000, China; and Mindray North America, Mahwah, New Jersey, USA; and Department of Surgical Oncology, Roswell Park Comprehensive Cancer Center, Buffalo, New York 14263, USA; Henry.Huang@mindray.com (H.H.)

\section{References}

1. DeLuca HF. Overview of general physiologic features and functions of vitamin D. Am J Clin Nutr. 2004;80(6 Suppl):1689S-96S.

2. Schmid A, Walther B. Natural vitamin D content in animal products. Adv Nutr. 2013;4(4):453-62.

3. Karras SN, Wagner CL, Castracane VD. Understanding vitamin D metabolism in pregnancy: From physiology to pathophysiology and clinical outcomes. Metabolism. 2018;86:112-123.

4. Colonese $F$, Laganà $A S$, Colonese $E$, et al. The pleiotropic effects of vitamin $D$ in gynaecological and obstetric diseases: an overview on a hot topic. Biomed Res Int. 2015;2015:986281.

5. Wang M, Chen Z, Hu Y, et al. The effects of vitamin D supplementation on glycemic control and maternal-neonatal outcomes in women with established gestational diabetes mellitus: A systematic review and meta-analysis. Clin Nutr. 2020 Dec 21:S0261-5614(20)30691-9.doi:

10.1016/j.clnu.2020.12.016.

6. Albahlol IA, Almaeen AH, Alduraywish AA, et al. Vitamin D Status and Pregnancy Complications: Serum 1,25-di-hydroxyl-Vitamin D and its Ratio to 25-hydroxy-Vitamin D are Superior Biomarkers than 25-hydroxy-Vitamin D. Int J Med Sci. 2020;17:3039-3048. doi: 10.7150/ijms.47807.

7. Vivanti AJ, Monier I, Salakos E, et al. Vitamin D and pregnancy outcomes: Overall results of the FEPED study. J Gynecol Obstet Hum Reprod. 2020;49:101883. doi: 10.1016/j.jogoh.2020.101883.

8. Gunasegaran P, Tahmina S, Daniel M, et al. Role of vitamin D-calcium supplementation on metabolic profile and oxidative stress in gestational diabetes mellitus: A randomized controlled trial. J Obstet Gynaecol Res. 2020 Dec 28. doi: 10.1111/jog.14629.

9. Ren Z, Zhe D, Li Z, et al. Study on the correlation and predictive value of serum pregnancy-associated plasma protein A, triglyceride and serum 25-hydroxyvitamin $D$ levels with gestational diabetes mellitus. World J Clin Cases. 2020;8:864-873.

10. Xu C, Ma HH, Wang Y. Maternal Early Pregnancy Plasma Concentration of 25-Hydroxyvitamin D and Risk of Gestational Diabetes Mellitus. Calcif Tissue Int. 2018;102:280-286.

11. Griew K, Nunn R, Fairbrother G, et al. Early pregnancy vitamin D deficiency and gestational diabetes: Exploring the link. Aust J Gen Pract. 2019;48:797-802. 
12. Hauta-Alus HH, Viljakainen HT, Holmlund-Suila EM, et al. Maternal vitamin D status, gestational diabetes and infant birth size. BMC Pregnancy Childbirth. 2017;17:420.

13. Arnold DL, Enquobahrie DA, Qiu C, et al. Early pregnancy maternal vitamin D concentrations and risk of gestational diabetes mellitus. Paediatr Perinat Epidemiol. 2015;29:200-210.

14. Schneuer FJ, Roberts CL, Guilbert C, et al. Effects of maternal serum 25-hydroxyvitamin D concentrations in the first trimester on subsequent pregnancy outcomes in an Australian population. Am J Clin Nutr. 2014;99:287-295.

15. Mclntyre HD, Catalano P, Zhang C, et al. Gestational diabetes mellitus. Nat Rev Dis Primers. 2019;5:47.

16. Damm P, Houshmand-Oeregaard A, Kelstrup L, et al. Gestational diabetes mellitus and long-term consequences for mother and offspring: a view from Denmark. Diabetologia. 2016;59:1396-1399.

17. Norman AW, Frankel JB, Heldt AM, et al. Vitamin D deficiency inhibits pancreatic secretion of insulin. Science. 1980;209:823-825.

18. Hu L, Zhang Y, Wang X, et al. Maternal Vitamin D Status and Risk of Gestational Diabetes: a MetaAnalysis. Cell Physiol Biochem. 2018;45:291-300.

19. Wilson RL, Leviton AJ, Leemaqz SY, et al. Vitamin D levels in an Australian and New Zealand cohort and the association with pregnancy outcome. BMC Pregnancy Childbirth. 2018;18:251.

20. Martínez-Domínguez SJ, Tajada M, Chedraui P, et al. Systematic review and meta-analysis of Spanish studies regarding the association between maternal 25-hydroxyvitamin D levels and perinatal outcomes. Gynecol Endocrinol. 2018;34:987-994.

21. Corcoy R, Mendoza LC, Simmons D, et al. The DALI vitamin D randomized controlled trial for gestational diabetes mellitus prevention: No major benefit shown besides vitamin D sufficiency. Clin Nutr. 2020;39:976-984.

22. Wen J, Kang C, Wang J, et al. Association of maternal serum 25 -hydroxyvitamin D concentrations in second and third trimester with risk of macrosomia. Sci Rep. 2018;8:6169.

23. Bodnar LM, Simhan HN, Catov JM, et al. Maternal vitamin D status and the risk of mild and severe preeclampsia. Epidemiology. 2014;25:207-214.

24. Palacios C, Trak-Fellermeier MA, Martinez RX, et al. Regimens of vitamin D supplementation for women during pregnancy. Cochrane Database Syst Rev. 2019;10:CD013446.

25. Mahmoud SZ, Saad AA, Mohieldein AH, et al. Serum level of 25-hydroxyvitamin D and obesity among early pregnant women. J Obstet Gynaecol Res. 2019;45:2338-2342. doi: 10.1111/jog.14114.

26. Shaheen S, Noor SS, Barakzai Q. Serum alkaline phosphatase screening for vitamin D deficiency states. J Coll Physicians Surg Pak. 2012;22:424-427.

27. Pulungan $A B$, Soesanti $F$, Tridjaja $B$, et al. Vitamin $D$ insufficiency and its contributing factors in primary school-aged children in Indonesia, a sun-rich country. Ann Pediatr Endocrinol Metab. 2021 Jan 7. doi: 10.6065/apem.2040132.066. 
28. Fernandez-Galan E, Bedini JL, Filella X. Analytical verification and method comparison of the ADVIA centaur intact parathyroid hormone assay. Clin Biochem. 2017; 50:1222-1227.

29. Kato $H$, Koga M, Kobayashi $H$, et al. Primary bone adult T cell lymphoma with multiple skeletal lesions and debilitating painful osteolysis: a case report. Osteoporos Int. 2020 Oct 31. doi: 10.1007/s00198-020-05720-6.

30. Saliba W, Awad K, Ron G, et al. The Effect of Vitamin D Supplementation on Thrombin Generation Assessed by the Calibrated Automated Thrombogram. Clin Appl Thromb Hemost. 2016;22:340-345.

\section{Tables}

Table 1 Basic information of study subjects

\begin{tabular}{|llllll|}
\hline Parameters & $\mathrm{n}$ & Min & Max & Mean & STDEV \\
\hline Age & 1516 & 18 & 46 & 29.42 & 4.58 \\
\hline BMI & 1516 & 13.80 & 40.90 & 26.76 & 3.25 \\
\hline BWON & 1516 & 940 & 4640 & 3182.32 & 452.04 \\
\hline Vitamin D & 1516 & 27.35 & 102.40 & 59.12 & 12.57 \\
\hline Glucose & 1516 & 3.40 & 6.30 & 4.31 & 0.34 \\
\hline ALT & 1489 & 2.00 & 481.00 & 13.16 & 16.07 \\
\hline ALP & 972 & 23.00 & 290.00 & 83.93 & 31.74 \\
\hline Pre-albumin & 951 & 63.00 & 349.00 & 222.83 & 32.85 \\
\hline Creatinine & 1466 & 27.00 & 96.00 & 47.14 & 7.21 \\
\hline Cystatin C & 323 & 0.47 & 1.84 & 0.96 & 0.22 \\
\hline Calcium & 1478 & 0.94 & 1.84 & 1.37 & 0.13 \\
\hline Magnesium & 1477 & 0.97 & 1.93 & 1.33 & 0.15 \\
\hline Bone density Z value & 924 & -3.06 & 1.34 & -0.51 & 0.49 \\
\hline D-Dimer & 1431 & 0.10 & 9.06 & 0.47 & 0.39 \\
\hline Fibrinogen & 1437 & 0.78 & 6.74 & 4.02 & 0.66 \\
\hline aPTT & 1437 & 2.00 & 48.40 & 36.28 & 3.07 \\
\hline TT & 1436 & 13.00 & 27.20 & 14.80 & 0.87 \\
\hline
\end{tabular}

BMI: body mass index; BWON: Body weight of newborn; aPTT: activated partial thromboplastin time; TT: thrombin time; Min: minimum; Max: maximum; STDEV: standard deviation 
Table 2 Correlation of diabetic status with serum vitamin D levels (independent-samples t-test)

\begin{tabular}{|lllllll|}
\hline & Glucose/ GDM & $\mathrm{n}$ & Average VD & STDEV & $\mathrm{t}$ & $p$ \\
\hline Fasting glucose & $<5.1$ & 1478 & 59.09 & 12.59 & -0.663 & 0.507 \\
\hline & $\geq 5.1$ & 38 & 60.46 & 11.48 & & \\
\hline Glucose of 1h GTT & $<10$ & 1358 & 59.27 & 12.59 & 1.350 & 0.178 \\
\hline Glucose of 2h GTT & $\geq 8.5$ & 158 & 57.86 & 12.36 & & \\
\hline & $\geq 8.5$ & 1342 & 59.30 & 12.56 & 1.491 & 0.136 \\
\hline GDM & 174 & 57.79 & 12.54 & & \\
\hline & No & 1252 & 59.27 & 12.58 & 0.940 & 0.347 \\
\hline
\end{tabular}

GDM: gestational diabetes mellitus; glucose (mmol/L); GTT: glucose tolerance test

Table 3 Correlation of serum vitamin D level with age and blood chemistry parameters (independentsamples t-test)

\begin{tabular}{|lllllll|}
\hline Parameters & & $\mathrm{n}$ & Average VD & STDEV & $\mathrm{t}$ & $p$ \\
\hline Age & $<=35$ & 1303 & 59.23 & 12.628 & 0.813 & 0.416 \\
\hline ALT & $>35$ & 211 & 58.47 & 12.245 & & \\
\hline Cystatin C & $<1.17$ & 274 & 59.12 & 12.45 & 1.355 & 0.176 \\
& $>=50$ & 17 & 56.53 & 12.19 & & \\
\hline Calcium & $>=1.17$ & 49 & 56.49 & 12.97 & & \\
\hline & $>1.42$ & 942 & 59.04 & 12.75 & -0.857 & 0.392 \\
\hline Magnesium & $>1.12$ & 71 & 58.33 & 11.38 & -0.634 & 0.526 \\
\hline & $>=1.12$ & 1406 & 59.31 & 12.68 & & \\
\hline
\end{tabular}

ALT: alanine aminotransferase (U/L); Cystatin C (mg/L); Calcium (mmol/L); Magnesium (mmol/L)

Table 4 Correlation of serum vitamin D with hematologic parameters (independent-samples t-test) 


\begin{tabular}{|lllllll|}
\hline Indices & Values & $\mathrm{n}$ & Mean & STDEV & $\mathrm{t}$ & $p$ \\
\hline RBC & $<3.5$ & 58 & 57.30 & 10.33 & -1.356 & 0.180 \\
& $>=3.5$ & 1439 & 59.20 & 12.66 & & \\
\hline HGB & $<=110$ & 232 & 58.91 & 12.28 & -0.282 & 0.778 \\
\hline WBC & $>110$ & 1265 & 59.16 & 12.63 & & \\
\hline & $>=10$ & 1081 & 59.08 & 12.63 & -0.239 & 0.811 \\
\hline Neu (\%) & $<70$ & 416 & 59.25 & 12.46 & & \\
\hline & $>=70$ & 1014 & 59.28 & 12.53 & & \\
\hline PLT & $100-300$ & 1244 & 59.31 & 12.61 & 1.298 & 0.195 \\
\hline & $>300$ & 253 & 58.19 & 12.39 & & \\
\hline
\end{tabular}

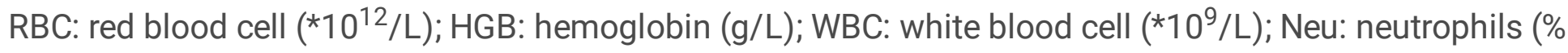
of WBC); PLT: platelet (*10\%/L)

Table 5a Correlation of serum vitamin D with parity (One-Way ANOVA)

\begin{tabular}{|lllllllll|}
\hline Parity & $\mathrm{n}$ & Mean & STDEV & SEM & Min & Max & $\mathrm{F}$ & $p$ \\
\hline 1 & 784 & 59.20 & 12.58 & 0.45 & 27.35 & 102.40 & 1.949 & 0.120 \\
\hline 2 & 652 & 58.66 & 12.44 & 0.49 & 27.43 & 92.50 & & \\
\hline 3 & 74 & 62.35 & 13.34 & 1.55 & 39.83 & 94.55 & & \\
\hline
\end{tabular}

Table $5 b$ Correlation of serum vitamin D with parity (One-Way ANOVA)

\begin{tabular}{|lllll|}
\hline (I) Parity & (J) Parity & Diff. of the means (I-J) & SEM & Sig. \\
\hline 1 & 2 & 0.54289 & 0.66543 & 0.415 \\
\hline 2 & 3 & -3.15422 & 1.52677 & 0.039 \\
\hline
\end{tabular}

SEM: standard error of the mean; Diff. of the means: difference of the means; Sig.: significance Table 6a Correlation of serum vitamin D and alkaline phosphatase activities (One-Way ANOVA) 


\begin{tabular}{|lcccccccc|}
\hline ALP & $\mathrm{n}$ & Mean & STDEV & SEM & Min & Max & F & $p$ \\
\hline$<45$ & 63 & 54.82 & 13.17 & 1.66 & 27.35 & 91.30 & 4.724 & 0.009 \\
\hline $45-125$ & 822 & 59.47 & 12.72 & 0.44 & 27.43 & 102.40 & & \\
\hline$>125$ & 87 & 60.90 & 11.57 & 1.24 & 34.78 & 91.15 & & \\
\hline
\end{tabular}

ALP: alkaline phosphatase activities

Table $\mathbf{6 b}$ Correlation of serum vitamin D and alkaline phosphatase activities (One-Way ANOVA)

\begin{tabular}{|lllll|}
\hline (I) ALP & (J) ALP & Diff. of the means (I-J) & SEM & Sig. \\
\hline$<45$ & $45-125$ & $-4.65378^{\star}$ & 1.65429 & 0.005 \\
\cline { 2 - 5 } & $>125$ & $-6.08375^{\star}$ & 2.09344 & 0.004 \\
\hline $45-125$ & $>125$ & -1.42998 & 1.42670 & 0.316 \\
\hline
\end{tabular}

Table 7 Correlation of serum vitamin D with bone density, body weight of newborns, coagulation, and other chemistry parameters (Pearson's correlation)

\begin{tabular}{|lllll|}
\hline \multicolumn{2}{|c}{$\mathrm{n}$} & & Vitamin D \\
\cline { 1 - 1 } Parameters & & & Pearson Correlation & Sig. (2-tailed) \\
\hline Bone density Z value & 924 & 0.019 & 0.555 \\
\hline Body weight of newborns & 1516 & -0.001 & 0.955 \\
\hline D-Dimer & 1431 & 0.035 & 0.180 \\
\hline Fibrinogen & 1437 & 0.043 & 0.107 \\
\hline TT & 1436 & -0.178 & 0.000 \\
\hline aPTT & 1437 & 0.052 & 0.047 \\
\hline Creatinine & 1466 & -0.071 & 0.006 \\
\hline Pre-albumin & 951 & 0.108 & 0.001 \\
\hline
\end{tabular}

TT: thrombin time; aPTT: activated partial thromboplastin time 